\title{
On Ribaucour transformations and applications to linear Weingarten surfaces
}

\author{
KETI TENENBLAT* \\ Departamento de Matemática, UnB, 70910-900 Brasília, DF, Brazil \\ Manuscript received on May 20, 2002; accepted for publication on July 15, 2002.
}

\begin{abstract}
We present a revised definition of a Ribaucour transformation for submanifolds of space forms, with flat normal bundle, motivated by the classical definition and by more recent extensions. The new definition provides a precise treatment of the geometric aspect of such transformations preserving lines of curvature and it can be applied to submanifolds whose principal curvatures have multiplicity bigger than one. Ribaucour transformations are applied as a method of obtaining linear Weingarten surfaces contained in Euclidean space, from a given such surface. Examples are included for minimal surfaces, constant mean curvature and linear Weingarten surfaces. The examples show the existence of complete hyperbolic linear Weingarten surfaces in Euclidean space.
\end{abstract}

Key words: Ribaucour transformations, linear Weingarten surfaces, minimal surfaces, constant mean curvature.

\section{INTRODUCTION}

This is an expository article which presents a new definition of a Ribaucour transformation and includes some of its applications. The revised definition was introduced in (Corro and Tenenblat 2002), motivated by a discussion of the classical definition of a Ribaucour transformation for hypersurfaces and some more recent extensions to submanifolds with higher codimension and flat normal bundle (Corro 1997, Dajczer and Tojeiro 2002). The new definition provides a precise treatment of the geometric aspect of such transformations preserving lines of curvature and it also extends Ribaucour transformations to submanifolds whose principal curvatures have multiplicity bigger than one.

Ribaucour transformations have a wide range of applications. In this paper, we will restrict ourselves mainly to the results obtained in (Corro et al. 2000, 2001). We will show how Ribaucour

\footnotetext{
*Member of Academia Brasileira de Ciências

E-mail: keti@mat.unb.br
} 
transformations can be applied as a method of constructing linear Weingarten surfaces contained in $R^{3}$, in particular minimal and constant mean curvature $(\mathrm{cmc})$ surfaces, from a given such surface. The transformation for minimal surfaces is related to producing embedded planar ends.

The method, when applied to the cylinder, produces complete $n$-bubble cmc and linear Weingarten surfaces. Moreover, it provides an unnexpected result. Namely, the existence of complete hyperbolic linear Weingarten surfaces immersed in $R^{3}$. It is well known, by Hilbert's theorem, that there are no complete surfaces of constant negative curvature immersed in $R^{3}$. Although such surfaces and hyperbolic linear Weingarten surfaces correspond to solutions of the sine-Gordon equation, the examples constructed in (Corro et al. 2001) show that there exist infinitely many complete hyperbolic linear Weingarten surfaces in $R^{3}$.

\section{RIBAUCOUR TRANSFORMATION}

The classical Ribaucour transformation relates diffeomorphic surfaces $M$ and $\tilde{M}$ of $R^{3}$ such that at corresponding points the normal lines intersect at an equidistant point. Moreover, the set of intersection points is also required to describe a surface of $R^{3}$ and the diffeomorphism to preserve the lines of curvature. The classical theory includes the case of hypersurfaces parametrized by lines of curvature, where the principal curvatures of both hypersurfaces have multiplicity one, although this is not stated clearly.

The extension to submanifolds of higher codimension is quite recent. One of the difficulties relies on extending the condition on the intersection of the normal lines. The first attempt was given in (Tojeiro 1991). In (Corro 1997) and (Dajczer and Tojeiro 2002), two distinct extensions were given for submanifolds, with flat normal bundle, parametrized by lines of curvature.

Definition 2.1 (Corro 1997). Two manifolds $M^{n}$ and $\tilde{M}^{n}$, contained in $R^{n+2}$, with flat normal bundle and parametrized by lines of curvature, are said to be related by a Ribaucour transformation if there exist a diffeomorphism $\psi: M \rightarrow \tilde{M}$, which preserves lines of curvature, a differentiable function $h: M \rightarrow R$ and unit normal vector fields $N, \tilde{N}$, parallel in the normal connection of $M$ and $\tilde{M}$, respectively, such that $\forall q \in M, q+h(q) N(q)=\psi(q)+h(q) \tilde{N}(\psi(q))$ and the subset $q+h(q) N(q)$ is $n$-dimensional.

Definition 2.2 (Dajczer And Tojeiro 2002). Two holonomic isometric immersions $f: M^{n} \rightarrow$ $R^{n+p}$ and $\tilde{f}: \tilde{M}^{n} \rightarrow R^{n+p}$ are said to be related by a Ribaucour transformation when there exist a curvature-lines-preserving diffeomorfism $\psi: M \rightarrow \tilde{M}$ with $|f-\tilde{f} \circ \psi| \neq 0$ everywhere, a vector bundle isometry $P: T_{f}^{\perp} M \rightarrow T_{\tilde{f}}^{\perp} \tilde{M}$ covering $\psi$, and a vector field $\zeta \in T_{f}^{\perp} M$ that is nowhere a principal curvature normal of $f$, such that a) $P(\xi)-\xi=<\xi, \zeta>(f-\tilde{f} \circ \psi)$ for all $\xi \in T_{f}^{\perp} M$; and b) $P$ is parallel, i.e. $P$ commutes with the normal connection.

The first definition is quite simple compared to the second one. The definition of (Tojeiro 1991) ommits from Definition 2.2 the requirement of a vector field $\zeta$ which is nowhere a principal curvature normal of the immersion. We also mention that Definition 2.2, proposed by Dajczer and Tojeiro, was used in (Brück et al. 2002). Before we relate these two definitions, we discuss 
some basic geometric aspects which motivated the new definition. We start by observing that the definitions above require a Ribaucour transformation to preserve all lines of curvature. This is one of the basic problems that will treated.

We first mention that, even in the case of surfaces in $R^{3}$, one has to fix a surface and then consider those associated to the given surface by Ribaucour tranformations (instead of considering two surfaces associated by the transformation). An easy example illustrates this observation. Consider in $R^{3}$ the following segments: $(1,0, t),(1+t, 0, t)$ and $(1+t, 0,0)$, where $t>0$. By rotating the segments arround the $x_{3}$ axis one gets a half cylinder, a truncated cone and the complement of a unit disc in the $x_{1}, x_{2}$ plane. Let $\psi$ be the diffeomorphism that to each point of the cylinder $(\cos \theta, \sin \theta, t)$ it associates the point $((1+t) \cos \theta,(1+t) \sin \theta, 0)$ on the plane. Then the truncated cone is the set of intersection of the normal lines and $\psi$ preserves the lines of curvature. However, one cannot say that the cylinder is associated to the planar region, since not all lines of curvature of the plane correspond to such curves on the cylinder.

\section{Preserving Lines of Curvature}

It is generally accepted that Ribaucour transformations preserve lines of curvature. In the classical theory and in both (Corro 1997) and (Dajczer and Tojeiro 2002), the definition is characterized essencially by the same integrable system of differential equations, whose solutions provide immersions locally associated by Ribaucour transformations to a given immersion. However, one can show (see Corollary 2.10 and also Corro et al. 1999) that this procedure does not always preserve multiplicity of principal curvatures. In such cases, the requirement of $\psi$ preserving all lines of curvature does not hold. This is due to the fact that the system of differential equations is a necessary condition for the existence of immersions associated to a given one by Ribaucour transformations, but it is not sufficient. Indeed one can show that given any hypersurface $M^{n}$ of $R^{n+1}$, which admits $n$ orthonormal principal direction vector fields, there exists a solution to the system of differential equations so that the associated hypersurface is an open subset of a hyperplane or a sphere (see Corollary 2.10).

In the new definition, the requirement of preserving lines of curvature is replaced by the requirement of preserving the lines of curvature corresponding to a fixed set of $n$ orthonormal vector fields of principal directions. In that case, the system of equations (which appear in [Corro 1997, Corro et al. 1999, Dajczer and Tojeiro 2002]) is indeed equivalent to the definition. Moreover, for submanifolds which admit principal curvatures with multiplicity bigger than one, in any dimension or codimension, the choice of distinct set of orthonormal principal directions may provide, by solving the system of equations, distinct families of submanifolds associated by Ribaucour transformations (see Remark 2.11).

\section{HolONOMIC SUBMANIFOLDS}

The classical theory and Definitions 2.1 and 2.2 require the submanifolds to be holonomic. (i.e. they admit a global parametrization by lines of curvature). However, by considering distinct such 
parametrizations (for hypersurfaces with admit principal curvatures with multiplicity bigger than one) one may obtain different associated submanifolds (see Remark 2.11). The nonholonomic case was considered in (Dajczer and Tojeiro 2001), with the same problem with respect to the transformation preserving all lines of curvature.

The new definition does not require the manifold to be holonomic. However, it requires the existence of $n$ orthomormal vector fields of principal directions globally defined, which will be preserved by the transformation. If the submanifold is parametrized by orthogonal lines of curvature, then the vector fields tangent to the coordinate curves are considered to be the principal directions that will be preserved. We observe that, for $n \geq 3$ the choice of a set of $n$ orthonormal vector fields of principal directions does not imply the existence of a local parametrization such that the coordinate curves are tangent to these vector fields, nor that the submanifold is holonomic.

\section{EXTENDing THE CONDition ON THE InTERSEction OF THE NoRMAL Lines}

Assuming that the submanifolds have flat normal bundle, while Definition 2.1 requires the existence of a unit vector field normal to each submanifold such that the corresponding lines intersect at an equidistant point, Definition 2.2 requires the existence of an isometry of the normal bundles such that the corresponding normal lines are parallel or intersect at an equidistant point.

The new definition requires the existence of a vector field normal to each submanifold such that at corresponding points the lines in these normal directions intersect at an equidistant point. It can be shown that this condition implies the existence of a correspondence between the normal bundles (resp. tangent bundles) such that the lines at corresponding normal (resp. tangent) vectors are parallel or intersect at an equidistant point. Moreover, the correspondence between the normal bundles can be chosen so that it is an isometry wich commutes with the normal connection.

\section{The Set of Equidistant Points}

The classical definitions and Definition 2.1 requires that the set of the intersections of the normal lines is an $n$-dimensional submanifold. The existence of a normal vector field, which is nowhere a principal curvature normal to the immersion in Definition 2.2, is equivalent to requiring the existence of a normal vector field for which the set of intersections of the corresponding normal lines is an $n$ dimensional submanifold.

In view of the aspects mentioned above, the revised definition is as follows:

Definition 2.3 (Corro And Tenenblat 2002). Let $M^{n}$ be a submanifold of $R^{n+p}$ with flat normal bundle. Assume there exist $e_{1}, \ldots, e_{n}$ orthonormal principal vector fields defined on $M$. A submanifold $\tilde{M}^{n} \subset R^{n+p}$, with flat normal bundle, is associated to $M$ by a Ribaucour transformation with respect to $e_{1}, \ldots, e_{n}$ if there exist a diffeomorphism $\psi: M \rightarrow \tilde{M}$, a differentiable function $h: M \rightarrow R$ and unit normal vector fields $N$ and $\tilde{N}$, parallel in the normal connection of $M$ and $\tilde{M}$ respectively, such that:

a) $q+h(q) N(q)=\psi(q)+h(q) \tilde{N} \psi(q), \forall q \in M$; 
b) the subset $q+h(q) N(q), q \in M$ is $n$-dimensional;

c) $d \psi\left(e_{i}\right)$ are orthogonal principal directions in $\tilde{M}$.

This transformation is invertible in the sense that there exist principal direction vector fields $\tilde{e}_{1}, \ldots, \tilde{e}_{n}$ on $\tilde{M}$ such that $M$ is associated to $\tilde{M}$ by a Ribaucour transformation with respect to these vector fields. One may consider the analogue local definition.

Definition 2.4. Let $M^{n}$ be a submanifold of $R^{n+p}$ with flat normal bundle. Assume there exist $e_{1}, \ldots, e_{n}$ orthonormal principal vector fields globally defined on M. A submanifold $\tilde{M}^{n}$ is locally associated to $M$ by Ribaucour transformations with respect to $e_{1}, \ldots, e_{n}$ if for any $\tilde{q} \in \tilde{M}$ there exists a neighborhood $\tilde{V}$ of $\tilde{q}$ in $\tilde{M}$ and an open subset $V \subset M$ such that $\tilde{V}$ is associated to $V$ by a Ribaucour transformation with respect to $e_{1}, \ldots, e_{n}$.

Similar definitions can be considered for immersions in $R^{n+p}$ and also for submanifolds and immersions in the sphere $S^{n+p}$ or the hyperbolic space $H^{n+p}$. In the latter cases one should replace the straight lines of conditions a) and b) by geodesics of the ambient space.

The above definition reduces to the classical case of surfaces in $R^{3}$ or hypersurfaces in $R^{n}$, parametrized by lines of curvature, whenever the principal curvatures of the associated submanifolds have multiplicity one. Moreover, it is equivalent to the system of differential equations which appeared in the papers mentioned previously.

The requirement of $\psi$ being a diffeomorphism implies that both manifolds are topologically equivalent. In general this is a very strong condition. Many interesting applications of this method (see section 3) show that in general one has immersions locally associated by Ribaucour transformations to a given one, even when both manifolds are complete. Moreover, the integrability of the corresponding system of differential equations imposes one to consider solutions on the universal covering of the given immmersion.

In what follows we consider a submanifold $M^{n}$ of $R^{n+p}$, with flat normal bundle. Let $e_{i}$, $1 \leq i \leq n$, be orthonormal principal vector fields on $M$ and let $N_{\alpha} 1 \leq \alpha \leq p$ be a an orthonormal frame normal to $M$ parallel in the normal connection. We denote by $\omega_{i}$ the one forms dual to the vector fields $e_{i}$ and by $\omega_{i j}, 1 \leq i, j \leq n$ the connection forms determined by $d \omega_{i}=\sum_{j \neq i} \omega_{j} \wedge \omega_{j i}$ and $\omega_{i j}+\omega_{j i}=0$ and the normal connection $\omega_{i \alpha}=<d e_{i}, N_{\alpha}>$. Since $e_{i}$ are principal directions, we have $d N_{\alpha}\left(e_{i}\right)=\lambda^{\alpha i} e_{i}$, i.e. $\omega_{i \alpha}=-\lambda^{\alpha i} \omega_{i}$. Details and proofs for the results in the remaining of this section can be found in (Corro and Tenenblat 2002).

THEOREM 2.5. Let $M^{n}$ be an immersed submanifold of $R^{n+p}$, whose normal bundle is flat and let $e_{i}, 1 \leq i \leq n$ be orthonormal principal vector fields defined on M. A submanifold $\tilde{M}^{n}$ is locally associated to $M$ by a Ribaucour transformation with respect to the set $e_{i}$, if and only if, for any $\tilde{q} \in \tilde{M}$ there exist parametrizations $\tilde{X}: U \subset R^{n} \rightarrow \tilde{M}$ and $X: U \rightarrow M$ such that $\tilde{q} \in \tilde{X}(U)$, a differentiable function $h: U \rightarrow R, a p \times p$ matrix function $B$ defined on $U$ and parallel orthonormal vector fields $N_{\alpha}, 1 \leq \alpha \leq p$, normal to $X(U)$ such that

$$
\tilde{X}=X+h\left(N_{1}-\tilde{N}_{1}\right) \text {, }
$$


where $\tilde{N}_{1}$ is a unit vector field normal to $\tilde{X}(U)$ given by

$$
\begin{aligned}
& \tilde{N}_{1}=\left(1-B^{11}\right) \sum_{i=1}^{n} Z^{i} e_{i}+\sum_{\gamma}^{p} B^{1 \gamma} N_{\gamma}, \\
& Z^{i}=\frac{d h\left(e_{i}\right)}{1+h \lambda^{1 i}} \quad \Delta=\sum_{i=1}^{n}\left(Z^{i}\right)^{2},
\end{aligned}
$$

$d N_{\alpha}\left(e_{i}\right)=\lambda^{\alpha i} e_{i}, \quad h$ and $B$ satisfy the differential equations

$$
\begin{gathered}
d Z^{j}\left(e_{i}\right)+\sum_{k=1}^{n} Z^{k} \omega_{k j}\left(e_{i}\right)-Z^{i} Z^{j} \lambda^{1 i}=0, \quad 1 \leq i \neq j \leq n, \\
B B^{t}+\Delta D D^{t}=I, \\
d B\left(e_{i}\right) B^{t}-B d B^{t}\left(e_{i}\right)+\Delta\left[d D\left(e_{i}\right) D^{t}-D d D^{t}\left(e_{i}\right)\right]+2 Z^{i}\left[B \Lambda^{i} D^{t}-D\left(\Lambda^{i}\right)^{t} B^{t}\right]=0
\end{gathered}
$$

where

$$
D^{t}=\left(1-B^{11},-B^{21}, \ldots,-B^{p 1}\right) \quad\left(\Lambda^{i}\right)^{t}=\left(\lambda^{1 i}, \ldots, \lambda^{p i}\right)
$$

In the theorem above, the associated parametrized manifold $\tilde{X}$ depends on a function $h$ and a matrix $B$ satisfying the differential equations (5) and (6). However, the expressions of the parametrization $\tilde{X}$ given by (1) and its normal vector field $\tilde{N}_{1}$ depend only on the first row of matrix $B$. The other rows of $B$ are related to fixing the unit vector fields $\tilde{N}_{\gamma}$, normal to $\tilde{X}$, for $\gamma \geq 2$.

If $\tilde{M}$ is associated to $M$ as in Definition 2.3, then one can show that for each point $\tilde{q} \in \tilde{M}$ and any unit vector normal (resp. tangent) to $\tilde{M}$ at $\tilde{q}$, there exists a unit vector, normal (resp. tangent) to $M$ at a corresponding point $q$, such that the lines in these directions are parallel or intersect at an equidistant point. One can also prove that there exists a matrix $B$, which satisfies (5) and (6), such that the correspondence between the normal bundles is an isometry which commutes with the normal connections (hence it satisfies the conditions of the Definition 2.2).

One can linearize the problem of obtaining the function $h$, by considering $h=\Omega / W_{1}$ and $\Omega_{i}$ defined by $\Omega^{i}=d \Omega\left(e_{i}\right)$. With this notation, equation (4) is equivalent to a linear system given in the following result.

Proposition 2.6. A function $h$ is a solution of (4) defined on a simply connected domain, if and 
only if, $h=\Omega / W_{1}$ where $W_{1}$ is a nonvanishing function and $\Omega, \Omega^{i}, W_{1}$ satisfy

$$
\begin{aligned}
d \Omega^{i}\left(e_{j}\right) & =\sum_{k=1}^{n} \Omega^{k} \omega_{i k}\left(e_{j}\right), \quad \text { for } i \neq j, \\
d \Omega & =\sum_{i=1}^{n} \Omega^{i} \omega_{i}, \\
d W_{1} & =-\sum_{i=1}^{n} \Omega^{i} \lambda^{1 i} \omega_{i} .
\end{aligned}
$$

Proposition 2.7. Equation (7) is the integrability condition for (8) and (9). Moreover, (7) implies that there exist functions $W_{\gamma}, 2 \leq \gamma \leq p$, defined on a simply connected domain such that

$$
d W_{\gamma}=-\sum_{i} \Omega^{i} \lambda^{\gamma i} \omega_{i}, \quad 2 \leq \gamma \leq p
$$

Proposition 2.8. Equations (7)-(10) are the integrability conditions, for the system of equations (5) and (6) for B. Moreover, for a given solution of (7)-(10) the matrix function

$$
B^{\alpha \beta}=\delta_{\alpha \beta}-2 \frac{W_{\alpha} W_{\beta}}{S}, \quad 1 \leq \alpha, \beta \leq p,
$$

where

$$
S=\sum_{j=1}^{n}\left(\Omega^{j}\right)^{2}+\sum_{\gamma=1}^{p}\left(W_{\gamma}\right)^{2}
$$

is a solution of (6).

As a consequence of Proposition 2.8, Theorem 2.5 can be rewritten as follows.

THEOREM 2.9. Let $M^{n}$ be an immersed submanifold of $R^{n+p}$, with flat normal bundle parametrized by $X: U \subset R^{n} \rightarrow M$. Assume $e_{i}, 1 \leq i \leq n$ are the principal directions, $N_{\gamma}, 1 \leq \gamma \leq p$, is a parallel orthonormal basis of the normal bundle of $X(U)$ and $\lambda^{\gamma i}$ the corresponding principal curvatures. A submanifold $\tilde{M}^{n}$ is locally associated to $M$ by a Ribaucour transformation with respect to $e_{i}$, if and only if, for each $\tilde{q} \in \tilde{M}$, there exist differentiable functions $W_{\gamma}, \Omega, \Omega^{i}: V \subset$ $U \rightarrow R$, defined on a simply connected domain $V$, which satisfy (7)-(10), such that, for some $1 \leq \alpha \leq p$

$$
W_{\alpha} S\left(W_{\alpha}+\lambda^{\alpha i} \Omega\right)\left(S-\Omega T^{i}\right) \neq 0, \quad 1 \leq i \leq n,
$$

where, $S$ is defined by (12),

$$
T^{i}=2\left(d \Omega^{i}\left(e_{i}\right)+\sum_{k} \Omega^{k} \omega_{k i}\left(e_{i}\right)-\sum_{\gamma=1}^{p} W_{\gamma} \lambda^{\gamma i}\right),
$$


and $\tilde{X}: V \subset R^{n} \rightarrow \tilde{M}$, is a parametrization of a neighborhood of $\tilde{q}$ in $\tilde{M}$ given by

$$
\tilde{X}=X-\frac{2 \Omega}{S}\left(\sum_{i=1}^{n} \Omega^{i} e_{i}-\sum_{\gamma=1}^{p} W_{\gamma} N_{\gamma}\right) .
$$

From now on, whenever we say that a submanifold $\tilde{M}$ is locally associated by a Ribaucour transformation to $M$ with respect to $e_{i}$, we are assuming that $e_{i}$ are orthonormal principal direction vector fields on $M$ and there are functions $\Omega^{i}, \Omega$ and $W_{\gamma}$, locally defined, satisfying the system (7)-(10).

The following result states that an $n$-dimensional sphere or a hyperplane can be locally associated by a Ribaucour transformation to any given hypersurface $M^{n} \subset R^{n+1}$, which admits $n$ orthonormal principal direction vector fields.

COROLlARY 2.10. Let $M^{n}$ be a hypersurface of $R^{n+1}$, that admits $n$ orthonormal principal direction vector fields $e_{i}$. The system of equations (7)-(9) with the additional algebraic condition

$$
S=b_{0} \Omega+b_{1} W
$$

where $S$ is defined by (12), is integrable for any real constants $b_{0}$ and $b_{1} \neq 0$. Moreover, any point of the locally associated hypersurface $\tilde{M}$ is umbilic, with principal curvatures equal to $b_{0} / b_{1}$. Hence $\tilde{M}$ is an open subset of a sphere (resp. hyperplane) if $b_{0} \neq 0\left(\right.$ resp. $\left.b_{0}=0\right)$.

This corollary shows that the system of equations (7)-(9) does not preserve multiplicity of principal curvatures. This fact had already been observed in (Corro et al. 1999).

We conclude this section with a remark which shows the effect of choosing distinct sets of orthonormal principal directions (or distinct parametrizations by lines of curvatures), when one applies Ribaucour transformations.

REMARK 2.11. Whenever the multiplicity of the principal curvatures of $M$ is bigger than one, the submanifolds $\tilde{M}$ associated by a Ribaucour transformation to $M$ may differ depending on the choice of the set of principal directions.

In fact, if we consider the parametrization $X\left(u_{1}, u_{2}\right)=\left(u_{1}, u_{2}, 0\right)$ of an open subset $U$ of the plane in $R^{3}$ and $e_{i}=X_{u_{i}}, i=1,2$, to be the unit tangent vectors, then the family of Dupin parametrized surfaces $\tilde{X}$, locally associated to $X$ by a Ribaucour transformation with respect to $e_{i}$ does not contain a parametrization of a torus. However, if we consider the open subset of the plane parametrized by $X\left(u_{1}, u_{2}\right)=\left(u_{1} \cos u_{2}, u_{1} \sin u_{2}, 0\right), 0<u_{1}<\infty, 0<u_{2}<2 \pi$. and the principal directions $\bar{e}_{1}=X_{u_{1}}$ and $\bar{e}_{2}=X_{u_{2}} / u_{1}$, then one can show that an open subset of a torus is locally associated to the plane by a Ribaucour transformation.

\section{APPLICATIONS TO LINEAR WEINGARTEN SURFACES}

We start mentioning a few facts about linear Weingarten surfaces. These are surfaces whose mean curvature $H$ and Gaussian curvature $K$ satisfy a linear relation $\alpha+\beta H+\gamma K=0$, where 
$\alpha, \beta, \gamma$ are real numbers. A linear Weingarten surface is said to be hyperbolic ( resp. elliptic) when $\Delta:=\beta^{2}-4 \alpha \gamma<0$ (resp. $\Delta>0$ ). The relation $\Delta=0$ characterizes the tubular surfaces. In particular, surfaces of constant negative Gaussian curvature are hyperbolic, while surfaces of constant mean curvature (including minimal) and constant positive curvature are elliptic. Hyperbolic (resp. elliptic) linear Weingarten surfaces correspond to solutions of the sine-Gordon equation (resp. elliptic sinh, cosh-Gordon equation) (see for example Tenenblat 1998).

Ribaucour transformations for constant Gaussian curvature and constant mean curvature (including minimal surfaces), were known since the beginning of last century (Bianchi 1927). However, they were applied for the first time to obtain minimal surfaces in (Corro et al. 2000). More recently, in (Corro et al. 2001), the method was extended to linear Weingarten surfaces, providing a unified version of the classical results.

We observe that linear Weingarten surfaces are locally parallel to surfaces of constant Gaussian curvature or to minimal surfaces. However, the Ribaucour transformations for these surfaces cannot be applied to produce complete linear Weingarten surfaces, since these parallel constructions in general produce curves of singularities.

THEOREM 3.1. Let $M$ be a regular surface of $R^{3}$, which admits orthonormal principal vector fields $e_{1}, e_{2}$. Let $\tilde{M}$ be a regular surface associated to $M$ by a Ribaucour transformation with respect to $e_{i}$, such that the function $h$ is not constant along the lines of curvature. Assume that the solutions $\Omega_{i}, \Omega$ and $W$ of (7)-(9) satisfy the additional relation

$$
S=2 c\left(\alpha \Omega^{2}+\beta \Omega W+\gamma W^{2}\right),
$$

where $S$ is defined by (12), $c \neq 0$ and $\alpha, \beta, \gamma$ are real constants. Then $\tilde{M}$ is a linear Weingarten satisfying $\alpha+\beta \tilde{H}+\gamma \tilde{K}=0$, if and only if $\alpha+\beta H+\gamma K=0$ holds for the surface $M$, where $K, H$ and $\tilde{K}, \tilde{H}$ are the Gaussian and mean curvatures of $M$ and $\tilde{M}$ respectively. Moreover, $\tilde{M}$ has no umbilic points, if and only if, $M$ has no umbilic points.

One can show that (7)-(9) with the additional condition (13) is integrable, whenever we start with a linear Weingarten surface. The solution is uniquely determined on a simply connected domain $U$, by any given initial condition satisfying (13). Moreover, whenever $\alpha \neq 0$, any solution of the system defined on $U$ is either identically zero and hence anihilates $S$ or else the function $S$ does not vanish on $U$.

If $M$ is a linear Weingarten surface locally parametrized by $X: U \subset R^{2} \rightarrow M \subset R^{3}$, then any linear Weingarten parametrized surface $\tilde{X}$, locally associated to $X$ by a Ribaucour transformation as above, is regular on

$$
\tilde{U}=\left\{\left(u_{1}, u_{2}\right) \in U ; T^{2}+2 T Q H+Q^{2} K \neq 0\right\}
$$

where $T=\alpha \Omega^{2}-\gamma W^{2}$ and $Q=2 \gamma \Omega W+\beta \Omega^{2}$.

Special cases of the above results include the minimal surfaces and the cmc surfaces. The cmc surfaces are obtained by considering $\alpha=-H \neq 0, \beta=1, \gamma=0$ and hence the algebraic 
condition (13) reduces to $S=2 c \Omega(-H \Omega+W)$, where $c$ satisfies the relation $c(c-2 H)>0$. For any nontrivial solution of the system (7)-(9) and (13), defined on a simply connected domain $U$, the function $S$ does not vanish. Hence, if $X: U \subset R^{2} \rightarrow R^{3}$ is a cmc suface, then a cmc surface $\tilde{X}$ associated to $X$ by Ribaucour transformation is regular on open subsets of $U$, where $X$ has no umbilic points.

The case of the minimal surfaces is obtained by considering $\alpha=0, \beta=1$, and $\gamma=0$ and the algebraic condition reduces to $S=2 c \Omega W$. One can show that the Ribaucour transfomations for minimal surfaces are related to producing embedded planar ends for the new associated minimal surfaces. In fact such ends are produced by the isolated zeros of $S$, where $\Omega$ does not vanish.

The reader is referred to (Corro et al. 2000, 2001) for proofs and details in the case of the minimal surfaces, linear Weingarten and cmc surfaces. In what follows, we first describe the families of minimal surfaces associated to Enneper surface and to the catenoid. Then the family of linear Weingarten surfaces associated by a Ribaucour transformation to the cylinder is discussed.

Proposition 3.2. Consider Enneper's surface parametrized by

$$
X\left(u_{1}, u_{2}\right)=\left(u_{1}-\frac{u_{1}^{3}}{3}+u_{1} u_{2}^{2}, u_{2}-\frac{u_{2}^{3}}{3}+u_{2} u_{1}^{2}, u_{1}^{2}-u_{2}^{2}\right)
$$

Excluding Enneper's surface, a parametrized surface $\tilde{X}\left(u_{1}, u_{2}\right)$ is a minimal surface locally associated to $X$ by a Ribaucour transformation as in Theorem 3.1, if and only if, up to a rigid motion of $R^{3}$,

$$
\tilde{X}=X+\frac{1}{c}\left(-u_{1}, u_{2}, 1\right)-\frac{1}{2 c(f+g)}\left(f^{\prime} X_{u_{1}}-g^{\prime} X_{u_{2}}\right)
$$

where $c$ is a positive real number,

$$
f=\delta \cosh \left(2 \sqrt{c} u_{1}+A\right) \quad g=\sin \left(2 \sqrt{c} u_{2}+B\right)
$$

where $\delta= \pm 1, A, B$ are real numbers and the functions $f$ and $g$ are defined in $R^{2} \backslash\left\{p_{k}, k \in Z\right\}$, where $p_{k}=\frac{1}{\sqrt{c}}(-A / 2,-\delta \pi / 4-B / 2+k \pi)$.

The family of minimal surfaces associated to Enneper's surface depends on three parameters. Each surface of this family has infinite total curvature and corresponds to a complete immersion of a sphere punctured at an infinite number of points, which are contained on a circle and accumulate at the pole. All except one of the infinite number of ends are embedded planar ends, whose positions are determined by the parameters. Figure 1 illustrates a minimal surface from the family described by (14). In this figure, one can see two views of a region which contains two planar ends of the surface $\tilde{X}$ for the constants $A=0, B=1$ and $c=1$.

Our next result describes the family of minimal surfaces associated to the catenoid by Ribaucour transformations. Depending on the value of the parameter $c$ of the Ribaucour transformation, the associated surface may have infinitely many embedded planar ends (see Callahan et al. 1989 for minimal surfaces with an infinite number of anular ends) or any finite number of embedded planar 

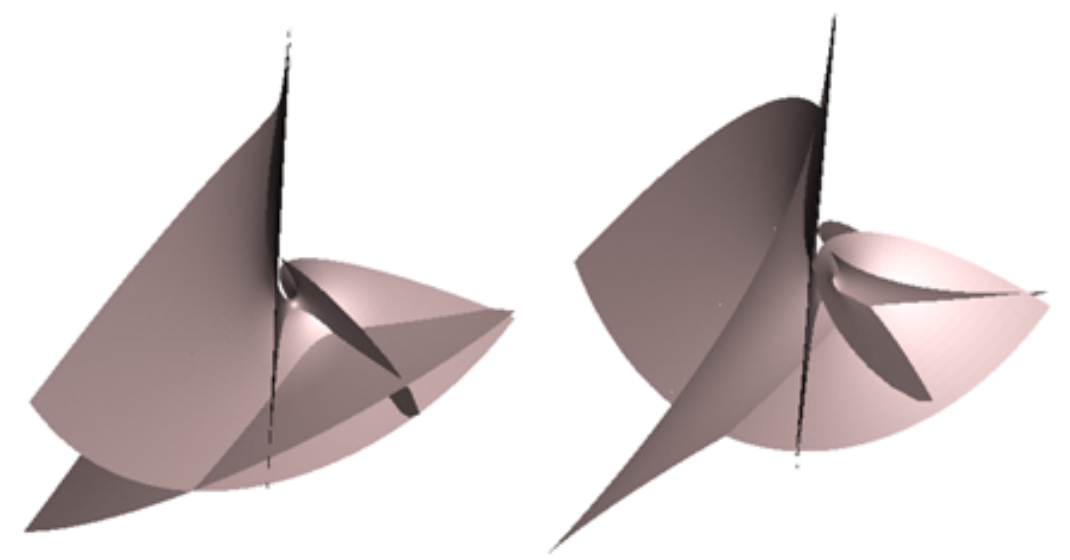

Fig. 1 - Minimal surface associated to Enneper's surface with $c=1, A=0, B=1$.

ends (see Jorge and Meeks 1983 for minimal surfaces with any finite number of catenoid ends). Moreover, each surface has one or two nonplanar ends. We point out that the family of minimal surfaces associated to the catenoid are of genus zero and contain a special class of 1-periodic surfaces.

Proposition 3.3. Consider the catenoid parametrized by

$$
X\left(u_{1}, u_{2}\right)=\left(\cos u_{2} \cosh u_{1}, \sin u_{2} \cosh u_{1}, u_{1}\right)
$$

Excluding the catenoid and up to rigid motions of $R^{3}$, a parametrized surface $\tilde{X}_{c}\left(u_{1}, u_{2}\right)$ is a minimal surface locally associated to $X$ by a Ribaucour transformation as in Theorem 3.1, if and only if,

$$
\tilde{X}_{c}=X-\frac{\cosh u_{1}}{c}\left(\cos u_{2}, \sin u_{2}, 0\right)+\frac{1}{c(f+g)}\left(f^{\prime} X_{u_{1}}-g^{\prime} X_{u_{2}}\right),
$$

where $c \neq 0, f\left(u_{1}\right)$ and $g\left(u_{2}\right)$ are given as follows:

a) if $c=1 / 2$, then $f=\frac{\left(c_{1} u_{1}+b_{1}\right)^{2}}{2 c_{1}}, \quad g=\frac{c_{1} u_{2}^{2}}{2}$, where $c_{1} \neq 0, b_{1} \in R$, and the function $\tilde{X}_{1 / 2}$ is defined on $R^{2} \backslash\left\{p_{1}\right\}$ with $p_{1}=-\frac{1}{c_{1}}\left(b_{1}, 0\right)$;

b) if $2 c-1>0$, then $f=\sin \left(A+\sqrt{2 c-1} u_{1}\right), \quad g= \pm \cosh \left(\sqrt{2 c-1} u_{2}\right), A \in R$ and the function $\tilde{X}_{c}$ is defined on $R^{2} \backslash\left\{p_{k}, k \in Z\right\}$, where $p_{k}=\frac{1}{\sqrt{2 c-1}}(\mp \pi / 2-A+2 k \pi, 0)$.

c) if $1-2 c>0$, then $f= \pm \cosh \left(A+\sqrt{1-2 c} u_{1}\right), \quad g=\sin \left(\sqrt{1-2 c} u_{2}\right), A \in R$ and the function $\tilde{X}_{c}$ is defined on $R^{2} \backslash\left\{p_{k}, k \in Z\right\}$, where $p_{k}=\frac{1}{\sqrt{1-2 c}}(-A, \mp \pi / 2+2 k \pi)$.

One can prove that any minimal surface $\tilde{X}_{c}$, locally associated to the catenoid, is complete. Its geometric properties are quite distinct, depending on the value of the parameter $c$. 
In particular, for special values of $c$, namely when $\sqrt{1-2 c}=n / m$ is a rational number, $n \neq m$, the associated family of minimal surfaces will be denoted by $\tilde{X}_{(n, m)}$. Any minimal surface of $\tilde{X}_{(n, m)}$ is 1-periodic in the variable $u_{2}$, has total curvature $-4 \pi(n+m)$ and it is an immersion of a sphere punctured at $n+2$ points: the two poles, corresponding to $u_{1} \rightarrow \pm \infty$, and $n$ points contained on a circle. Its Gauss map $\tilde{N}$ extends to the $n+2$ points. The surface has $n$ embedded planar ends and two ends $\mathcal{F}^{ \pm}$, of geometric index $m$, corresponding to $u_{1} \rightarrow \pm \infty$, that grow asymptotically as the ends of the catenoid. In particular, $\mathcal{F}^{ \pm}$are embedded catenoid ends, if and only if $m=1$. Figure 2 contains several examples of such surfaces.

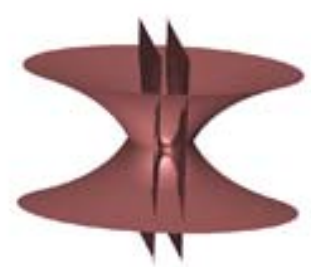

a)

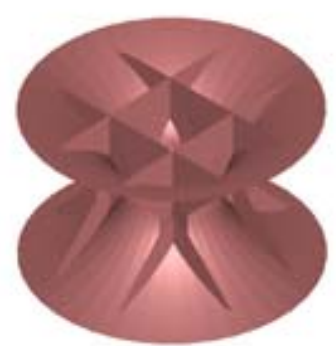

d)

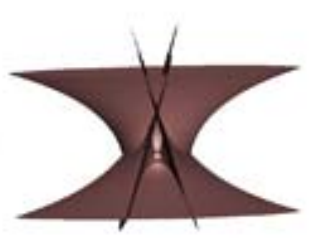

b)

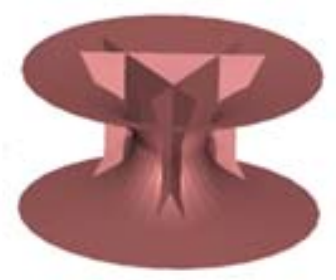

c)

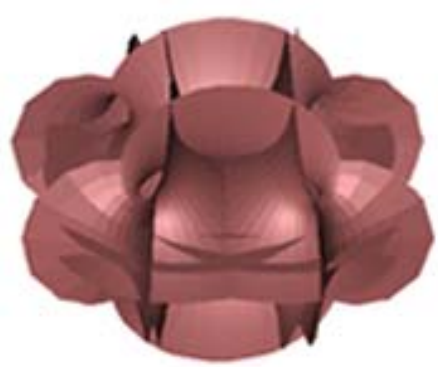

e)

Fig. 2 - Complete 1-periodic minimal surfaces $\tilde{X}_{(n, m)}$ associated to the catenoid by Ribaucour transformations. Each surface has $n$ planar ends and two ends of geometric index $m$. a) $n=2, m=1, A=0$; b) $n=2, m=1, A=1 / 2$; c) $n=3, m=1$, $A=0$; d) $n=4, m=1, A=0$; e) $n=4, m=3, A=0$.

Whenever $c$ is such that $2 c-1 \geq 0$ or $2 c-1<0$ and $\sqrt{1-2 c}$ is not a rational number, then $\tilde{X}_{c}$ is a family of complete minimal surfaces which have infinite total curvature and they are not periodic in any variable. Any surface of the family $\tilde{X}_{c}$ is an immersion of a sphere punctured at two points if $2 c-1=0$ and punctured at an infinite number of points contained on a circle, otherwise. Its normal map $\tilde{N}$ extends to the points $p_{k}$ described in Proposition 3.3. Moreover, the ends of any such surface are planar except the end corresponding to $|u| \rightarrow \infty$.

We observe that whenever $c=1 / 2$ any surface of $\tilde{X}_{1 / 2}$ is an immersed minimal surface with two ends. One of them, corresponding to $p_{1}$, is planar and the other one, corresponding to the pole, is not embedded. 
The families of minimal surfaces, associated to the catenoid depend on two parameters. While the geometric properties of the surfaces are determined by $c$, the position of the planar ends is determined by the other parameter (see Figure 2 a) and b)).

Our next application provides a two-parameter family of linear Weingarten surfaces,

Proposition 3.4. Consider the cylinder parametrized by

$$
X\left(u_{1}, u_{2}\right)=\left(\cos \left(u_{2}\right), \sin \left(u_{2}\right), u_{1}\right) \quad\left(u_{1}, u_{2}\right) \in R^{2}
$$

as a linear Weingarten surface satisfying $-1 / 2+H+\gamma K=0$. A parametrized surface is a linear Weingarten surface locally associated to $X$ by a Ribaucour transformation as in Theorem 3.1, if and only if, it is given by

$$
\tilde{X}_{c \gamma}=X-\frac{2(f+g)}{c\left[(2 \gamma+1) g^{2}-f^{2}\right]}\left(f^{\prime} X_{u_{1}}+g^{\prime} X_{u_{2}}-g N\right),
$$

where $N$ is the inner unit normal vector field of the cylinder, $c \neq 0$ and $\gamma$ are real constants, such that

$$
\xi(c, \gamma)=1-c(2 \gamma+1)
$$

and $c$ are not simultaneously positive, and $f\left(u_{1}\right), g\left(u_{2}\right)$ are solutions of the equations $f^{\prime \prime}+c f=$ $0, \quad g^{\prime \prime}+\xi g=0$, with initial conditions satisfying

$$
\left(\left(f^{\prime}\right)^{2}+\left(g^{\prime}\right)^{2}+\xi g^{2}+c f^{2}\right)\left(u_{1}^{0}, u_{2}^{0}\right)=0 .
$$

Moreover, $\tilde{X}_{c \gamma}$ is a regular surface defined on the subset of $U \subset R^{2}$ where

$$
\left((f+g)^{2}+2 \gamma g^{2}\right)\left(f^{2}+2(2 \gamma+1) f g+(2 \gamma+1) g^{2}\right) \neq 0 .
$$

The linear Weingarten surfaces $\tilde{X}_{c \gamma}$ associated to the cylinder and parametrized by (15) (excluding the cylinder), have curves of singularity if $c \xi \geq 0$. Moreover, if $c \xi<0$ then, up to rigid motions of $R^{3}$, the surface $\tilde{X}_{c \gamma}$ is determined by the functions

$$
\begin{array}{lrl}
f=\varepsilon_{1} \sqrt{|\xi|} \sin \left(\sqrt{c} u_{1}\right) & g=\varepsilon_{2} \sqrt{c} \cosh \left(\sqrt{|\xi|} u_{2}\right) & \text { if } c>0, \xi<0 \\
f=\varepsilon_{1} \sqrt{\xi} \cosh \left(\sqrt{|c|} u_{1}\right) & g=\varepsilon_{2} \sqrt{|c|} \sin \left(\sqrt{\xi} u_{2}\right) & \text { if } c<0, \xi>0
\end{array}
$$

where $\varepsilon_{i}= \pm 1, c \neq 0$ and $\gamma$ are real numbers and $\xi(c, \gamma)$ is defined by (16).

One can show that the surface $\tilde{X}_{c \gamma}$ is complete, if and only if, $c \xi(c, \gamma)<0$ and the pair $(c, \gamma)$ belongs to a region $\mathcal{D} \subset R^{2}$ with two connected components described in Figure 3 . The functions which determine the components of $\mathcal{D}$ are defined by

$$
\begin{aligned}
& h_{1}(c, \gamma)=2 c(2 \gamma+1)(\sqrt{2 \gamma(2 \gamma+1)}-2 \gamma)-1 \\
& h_{2}(c, \gamma)=2 c(\sqrt{2|\gamma|}+2 \gamma)-1 \\
& h_{3}(c, \gamma)=-2 c(2 \gamma+1)(\sqrt{2 \gamma(2 \gamma+1)}+2 \gamma)-1 \\
& h_{4}(c, \gamma)=2 c(\sqrt{2|\gamma|}-2 \gamma)+1 \\
& h_{5}(c, \gamma)=-2 c(2 \gamma+1)(\sqrt{2 \gamma(2 \gamma+1)}-2 \gamma)+1
\end{aligned}
$$




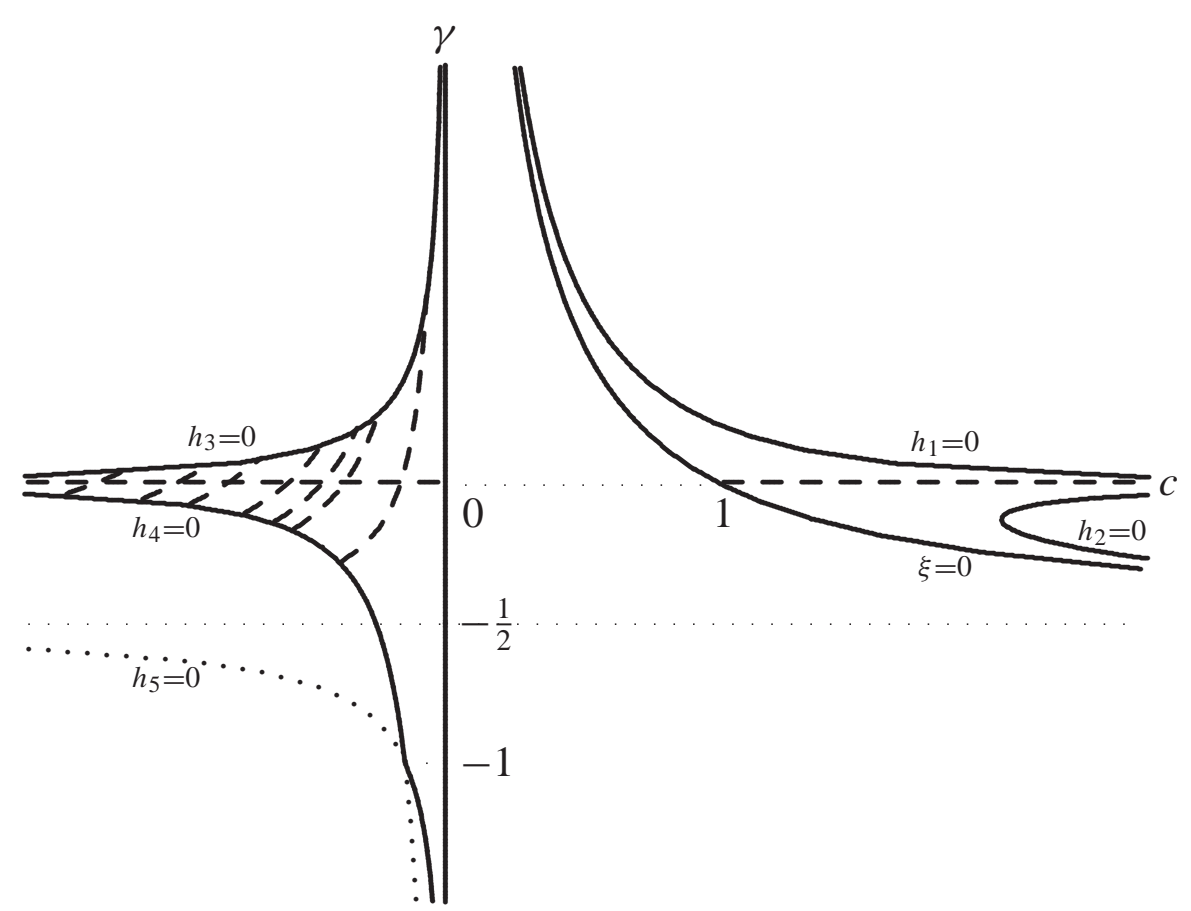

Fig. 3 - Any pair $(c, \gamma)$, in each of the two connected components, generates a complete linear Weingarten surface and it is cmc when $\gamma=0 .(c, \gamma)$ on the dashed curves in the left region generate 1-periodic $n$-bubble surfaces with two ends of geometric index $m$.

If $c<0$ and $\sqrt{\xi(c, \gamma)}=n / m$ is an irreducible rational number, then the linear Weingarten surface $\tilde{X}_{c \gamma}$ is periodic in the variable $u_{2}$ and hence it is an immersion of a cylinder into $R^{3}$. One proves that the immersed surface has two ends of geometric index $m$ and $n$ isolated points of maximum (respectively minimum) for the Gaussian curvature. Moreover, the total curvature of $\tilde{X}_{c \gamma}$ is zero, while its total absolute curvature is $8 \pi n$. The ends are embedded if and only if $m=1$; in this case they are cylindrical ends.

If $c>0$ or $c<0$ and $\sqrt{\xi}$ is not a rational number then the linear Weingarten surface, $\tilde{X}_{c \gamma}$, associated to the cylinder is not periodic in any variable. It is an immersion of $R^{2}$ into $R^{3}$ with an infinite number of isolated critical points of its Gaussian curvature.

One can also show that the complete linear Weingarten surfaces $\tilde{X}_{c \gamma}$ are asymptotically close to the cylinder. We observe that the surfaces given by $\tilde{X}_{c \gamma}$ are tubular surfaces when $\gamma=-1 / 2$, since they satisfy $\Delta=\beta^{2}-4 \alpha \gamma=0$. Figure 4 illustrates several examples of this family of surfaces.

We point out that the surfaces $\tilde{X}_{c \gamma}$, where $c<0$ and $\gamma<-1 / 2$, are hyperbolic since $\Delta<0$. Therefore, they show that there exist complete hyperbolic linear Weingarten surfaces immersed in $R^{3}$, although there are no such surfaces with constant negative curvature (Hilbert's theorem).

The class of linear Weingarten surfaces $\tilde{X}_{c \gamma}$ contains a family of $1 / 2 \mathrm{cmc}$-surfaces obtained 


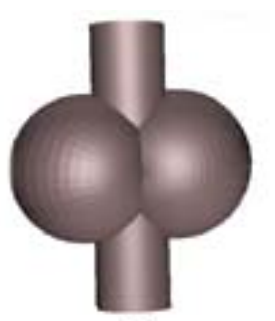

a)

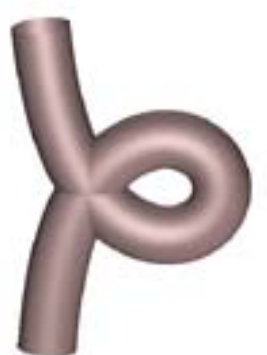

d)

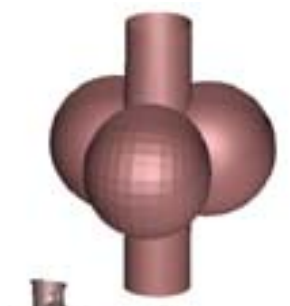

b)

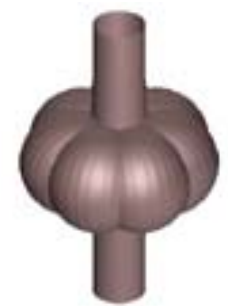

c)

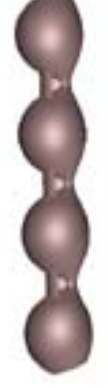

e)

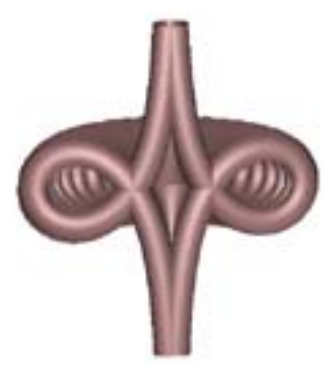

f)

Fig. 4 - Complete Weingarten surfaces $\tilde{X}_{c \gamma}$ which satisfy the relation $-1 / 2+H+\gamma K=0$ and are associated to the cylinder by Ribaucour transformations. a) 1-periodic cmc surface $\gamma=0$ and $\sqrt{1-c}=2$; b) 1-periodic cmc-surface for which $\gamma=0, \sqrt{1-c(2 \gamma+1)}=$ $3 / 2$; c) 1-periodic Weingarten surface, $\gamma=0.15, \sqrt{1-c(2 \gamma+1)}=7 / 5$; d) tubular surface, $\gamma=-1 / 2, c=-0.1$; e) cmc surface (not periodic) where $c=1.5$ and $\gamma=0$; f) part of a complete hyperbolic surface.

by considering $\gamma=0$. Whenever $c<0$, these cmc-surfaces have one spherical family of curvature lines. Such surfaces are called of Enneper type by Wente (Wente 1992). By choosing $\sqrt{1-c}=$ $n / m$, an irreducible number, one gets $n$-bubble cmc-surfaces. These surfaces were first described by Sievert (Sievert 1886) for $n=2$ (see also (Pinkal and Sterling 1989)) and their existence was proved later in (Große-Brauckmann 1993) and (Sterling and Wente 1993). For other values of $c$ the cmc-surfaces are not periodic in any variable.

Similarly, by using Ribaucour transformations one can obtain families of cmc surfaces associated to the Delaunay surfaces. By restricting the range of the parameter $c$ of the Ribaucour transformation, one gets families of complete cmc surfaces. For special values of $c$ one gets periodic surfaces in one variable otherwise the surfaces are not periodic in any variable. Details and proofs for the results on linear Weingarten surfaces and cmc-surfaces can be found in (Corro et al. 2001).

\section{ACKNOWLEDGMENTS}

The author's work is partially supported by CNPq. 


\section{RESUMO}

Apresentamos uma definição de tranformação de Ribaucour revisada, para subvariedades de formas espaciais com fibrado normal plano, motivados pela definição clássica e pelas extensões mais recentes. A nova definição fornece um tratamento preciso do aspecto geométrico de tais transformações preservarem linhas de curvatura e pode ser aplicada a subvariedades cujas curvaturas principais têm multiplicidade maior que um. Transformações de Ribaucour são aplicadas como um método para obtenção de superfícies de Weingarten lineares, contidas no espaço Euclideano, a partir de uma dada superfície deste tipo. Exemplos são incluidos para superfícies mínimas, superfícies de curvatura média constante e superfícies linear Weingarten. Os exemplos mostram a existência de superfícies linear Weingarten, hiperbólicas, completas no espaço Euclideano.

Palavras-chave: transformações de Ribaucour, superfícies de Weingarten lineares, superfícies mínimas, curvatura média constante.

\section{REFERENCES}

Bianchi L. 1927. Lezioni di Geometria Differenciale, Bologna Nicola Zanichelli Ed.

Brück M, Du X, Park J and Terng C-L. 2002. The submanifold geometries associated to Grassmannian systems. Mem Amer Math Soc 735.

Callahan M, Hoffman D and Meeks III, W. 1989. Embedded minimal surfaces with an infinite number of ends. Invent Math 96: 459-505.

Corro AV. 1997. Transformações entre subvariedades de Dupin. PhD thesis, Univ de Brasília.

Corro AV, Ferreira W and Tenenblat K. 1999. On Ribaucour transformation for hypersurfaces. Mat Contemp 17: 137-160.

Corro AV, Ferreira W and Tenenblat K. 2000. Minimal surfaces obtained by Ribaucour transformations. Preprint. Geometria Dedicata, (to appear).

Corro AV, Ferreira W and Tenenblat K. 2001. Ribaucour transformations for $\mathrm{cmc}$ and linear Weingarten surfaces. Preprint. Universidade de Brasília.

Corro AV and Tenenblat K. 2002. Ribaucour transformations revisited. Preprint. Universidade de Brasília.

Dajczer M And Tojeiro R. 2002. An extension of the classical Ribaucour transformation. Proc London Math Soc 85: 211-232.

Dajczer M And Tojeiro R. 2001. Commuting Codazzi tensors and the Ribaucour transformation for submanifolds. Preprint.

Große-Brauckmann, K. 1993. New surfaces of constant mean curvature. Math Zeit 214: 527-565.

Jorge LP AND MeEKs III W. 1983. The topology of complete minimal surfaces of finite total Gaussian curvature. Topology 2: 203-221. 
Pinkal U AND Sterling I. 1989. On classification of constant mean curvature mean tori. Ann Math 130: 407-451.

SIEvert H. 1886. Über die Zentralflächen der Enneperschen Flächen konstanten Krümmungsmasses, Diss. Tübingen.

Sterling I ANd Wente HC. 1993. Existence and classification of cmc multibubbleton of finite and infinite type. Indiana Univ Math J 42: 1239-1266.

Tenenblat K. 1998. Transformations of manifolds and applications to differential equations, Addison Wesley Longman, Pitman Monographs and Surveys in Pure and Applied Mathematics \# 93.

ToJeIRo R. 1991. Imersões isométricas entre espaços de curvatura constante. PhD thesis, IMPA.

Wente HC. 1992. Constant mean curvature immersions of Enneper type. Memoirs Amer Math Soc 478. 